Proceedings of the 2010 Winter Simulation Conference

B. Johansson, S. Jain, J. Montoya-Torres, J. Hugan, and E. Yücesan, eds.

\title{
BI-CRITERIA ANALYSIS OF AMBULANCE DIVERSION POLICIES
}

\author{
Adrian Ramirez Nafarrate \\ John W. Fowler \\ Teresa $\mathrm{Wu}$ \\ Arizona State University \\ 699 S. Mill Avenue, Suite 553 \\ Tempe, AZ 85281, USA
}

\begin{abstract}
Overcrowding episodes in the Emergency Departments (EDs) of the United States and their consequences have received considerable attention by the media and the medical community. One of these consequences is ambulance diversion (AD), which is adopted as a solution to relieve congestion. Current practices on $\mathrm{AD}$ decisions are largely dependent on human expertise, thus they tend to be subjective. This paper develops a simulation model for ED to study the impact of AD policies based on one of the following main ED state variables: the number of patients waiting, the number of patients boarding and the number of available beds in the inpatient unit. The objective is to analyze the impact of AD on the ED performance considering two criteria: patient average waiting time and percentage of time spent on diversion. Results show that there exist significant differences based on the variables chosen to design the policy and the criterion to reevaluate the AD status. This insight can be used to assist ED managers in making AD decisions to achieve better quality of healthcare service.
\end{abstract}

\section{INTRODUCTION}

Saturation and overcrowding episodes in ED's across the United States have been discussed by the media, healthcare organizations, government agencies, the medical community as well as other professional and academic organizations. Problems such as long waiting times, patients leaving without treatment (LWOT) and long boarding times have been analyzed using methods such as simulation and queuing formulations. This paper explores the use of simulation to study the decision of diverting ambulances to other facilities.

The General Accounting Office (US GAO 2003) submitted a report to the US Senate in 2003 highlighting some issues found in ED's. This report mentioned that about $10 \%$ of the emergency care providers that participated in the study spent more than $20 \%$ of the time on diversion during fiscal year 2001 . Furthermore, more than two thirds of the participants went on diversion at least once per year. Another study conducted in 2003 and published by the National Health Statistics Report, showed that the mean annual hours on diversion of ED's that reported any diversion was 403.9 hours (CDC 2006).

Since then, AD has been widely discussed in media and scholar publications. The AD decision which leads to potential delays for treatment due to longer transportation times, has been seriously criticized. Many local governments thus have prohibited going on AD status (CDC 2006). However, the "no ambulance diversion" policies have affected the ED performance negatively. For instance, hospitals located in Massachusetts have seen higher waiting times and a large number of patients boarding in inappropriate areas (Massachusetts Nurse Newsletter 2009).

In general, specialists agree that $\mathrm{AD}$ is a matter of public health and its implementation should be linked with clinical outcomes, patient and provider satisfaction, quality-of-life measures, economic measures and quality management initiatives (Asplin 2003). Therefore, this paper proposes the use of model- 


\section{Ramirez, Fowler and Wu}

ing methods to quantitatively analyze the effectiveness of the AD decision with multiple criteria being considered.

In this paper, a discrete-event simulation model of an ED is built to analyze policies that trigger the diversion status based on a single threshold. The thresholds are related to the main state variables of the $\mathrm{ED}$, which also are the main causes that practitioners take into account for diversion decisions. These variables are: the number of patients waiting in the ED, the number of patients boarding and the number of available beds in the inpatient units. The decision outcomes are assessed based on two criteria: patient average waiting time and percentage of time spent on diversion. Design of Experiments is first employed to select the appropriate levels for the policies and then bi-criteria Pareto analysis and simultaneous confidence ellipses are applied to study the trade off existing in the implementation of AD.

The rest of the paper reviews the literature regarding AD in Section 2, the proposed study is described in detail in Section 3, the analysis of the results is presented in Section 4 and finally the conclusions and future research are presented in Section 5.

\section{LITERATURE REVIEW}

Quantitative assessment for AD decision has been studied less than other issues related to ED (e.g., waiting time and staffing levels). This might be due to the complexity, the dependency on the local conditions and the preference for searching alternative solutions to overcrowding in AD decisions.

Simulation, queuing analysis and game theory are some commonly used modeling tools to analyze the impact of AD. Conclusions about this topic are varied and comprise multiple aspects. For instance, it has been found that the patient length of stay has a positive correlation with the probability of going on diversion (Kolker 2008). The configuration of the ED is also a significant factor on the diversion performance, for instance, design of experiments applied in a simulation model shows that a fast-track area to treat the least urgent patients could reduce the time spent on diversion status (Ramirez et al., 2009). Queuing analysis has been applied to obtain equations that measure the performance of an ED, which goes on diversion based on a policy of minimum-maximum on the number of patients waiting (Ramirez et al., 2010a). Game theory has been proposed to study the reciprocal effect found in a system of ED's that go on diversion. According to this analysis, there is a need of an external agent that regulates AD in a system of several hospitals (Hagtvedt, et al. 2009).

Despite the scarcity of mathematical assessment of the situation, AD has been widely discussed by the medical community. Important remarks found on these publications are the common factors that influence diverting ambulances. It has been found that diversion decision are made due to three main reasons: there is a high number of patients waiting in the ED, there is a high number of patients boarding or there is a lack of available beds in the inpatient units (Asplin 2003, CDC 2006). Nevertheless, to the best of our knowledge, quantitatively assessing the impact of these variables on an AD policy has not been done.

This paper proposes methods to analyze the performance of $\mathrm{AD}$ decisions on these variables in terms of mean performance and variability graphically. Two performance criteria are considered: (1) accessibility to emergency care, represented by percentage of time on diversion and (2) timely service, represented by the average patient waiting time.

\section{PROPOSED STUDY}

First, a simulation model representing a hypothetical ED that is representative of many emergency care facilities across the United States is developed. This model is adapted to conduct experiments for the chosen $\mathrm{AD}$ policy, which have a single threshold that triggers the diversion state. Then, continuous or periodic evaluation of the state of the system is used to re-evaluate or remove the diversion status. Design of experiments is used to define the threshold levels for all the AD policies. During the execution of the simulation model, output data regarding time on diversion and average patient waiting time is collected. Finally, Pareto analysis is applied to study and observe the main differences among the mean performance 
of the policies and simultaneous confidence ellipses are constructed to study the variability across policies. Figure 1 depicts the framework presented on this paper.

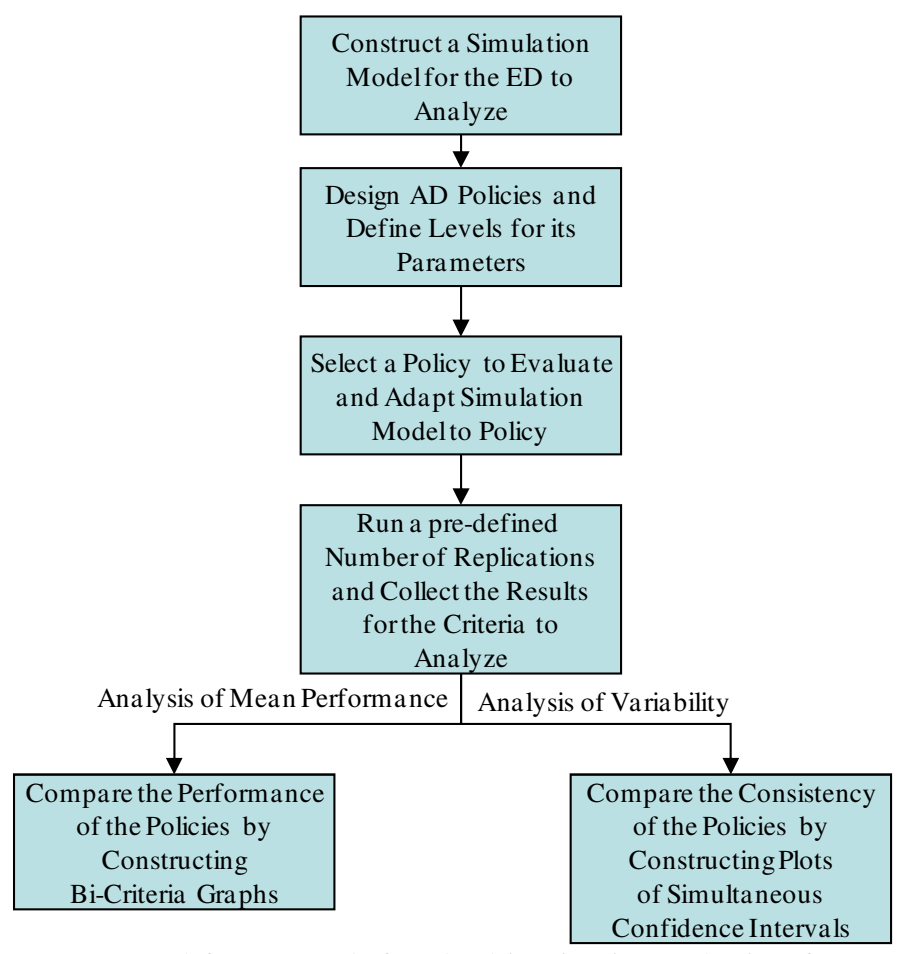

Figure 1: Proposed framework for the bi-criteria analysis of AD policies

\subsection{Simulation Model}

Figure 2 illustrates the simulation model which includes one ED and one Inpatient Unit (IP).

The ED receives patients arriving by ambulance or walking in. Upon arrival, the patients are classified to one out of five acuity levels. Patients of Level 1 are considered to have the most serious problems, while patients of Level 5 are the non-urgent patients. If the diversion status is on, any entity representing ambulance patients arriving in that period will be destroyed. If the diversion status is off and all the beds in the ED are occupied, the patients need to wait in a line for treatment. Treatment is only provided when patients are able to get a bed. The beds in the ED and in the IP unit are the only resources modeled.

Beds are assigned to patients based on a priority given by the acuity level, with the most acute patients receiving the highest priority. The mean treatment time depends on the acuity level as well. If a patient remains in the ED for a long time without starting treatment in a bed, then that entity will be removed of the simulation, representing an LWOT patient.

After completing treatment, the patients can be either discharged or admitted to the hospital. Therefore, the IP unit receives patients from direct admission and admissions from the ED. The treatment time in the IP unit depends on the origin of the patient. After completing treatment in the IP unit, the patients are discharged. If a patient from the ED needs to be admitted into the hospital, but there is not any available bed in the IP unit, then the patient remains in the ED occupying the bed and blocking the flow of other patients, this is referred to as boarding. 


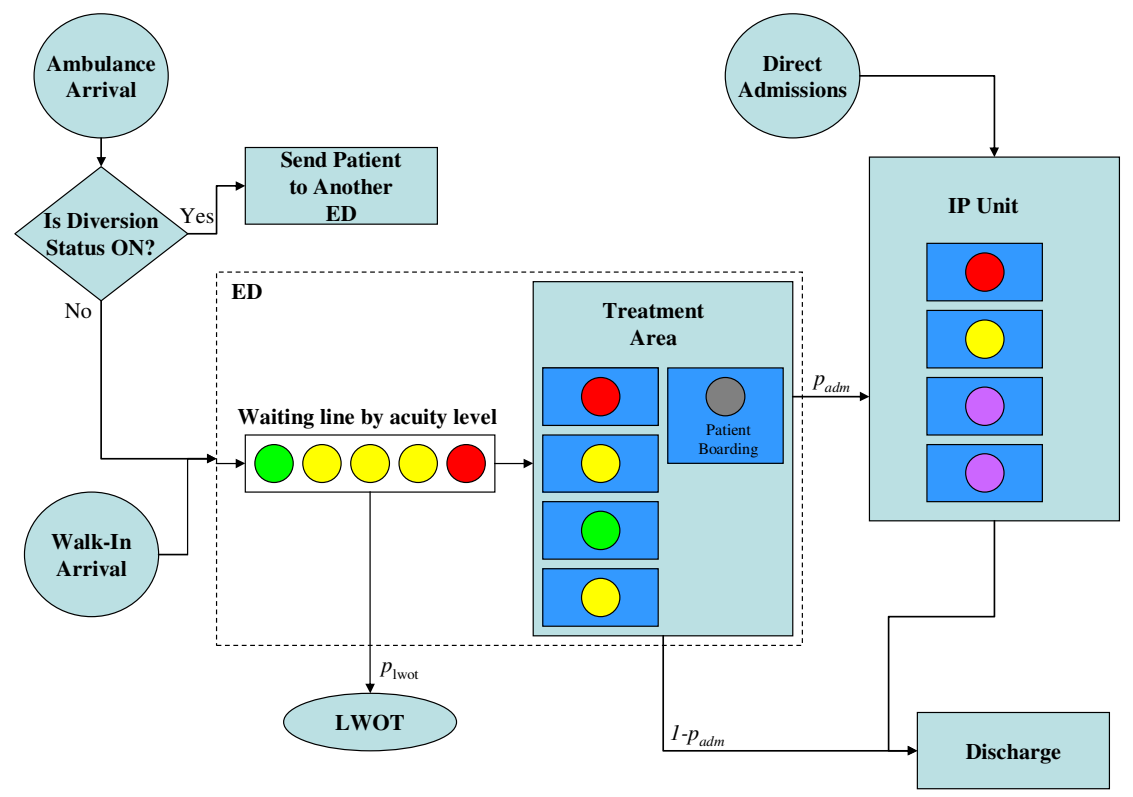

Figure 2: Logic of the simulation model

\subsection{Input Data}

The input data used in this model intends to be representative from emergency care providers and to capture the main dynamics seen in ED's across the United States. For instance, the arrival pattern exhibited in the ED has been highlighted by different publications (Cochran and Roche 2009; CDC 2008; Springer's International Series 2006; Miller, Ferrin and Shahi 2009). It is assumed in this paper that arrivals follow a Poisson process with a mean arrival rate depending on the arrival mode and time of the day. The rates shown in Figure 3 are obtained from a publication of an analysis of a provider in Arizona (Cochran and Roche 2009). Note that $15 \%$ of all the arrivals to the ED correspond to ambulance arrivals; this percentage is consistent with the national average of $15.4 \%$ (CDC 2008).

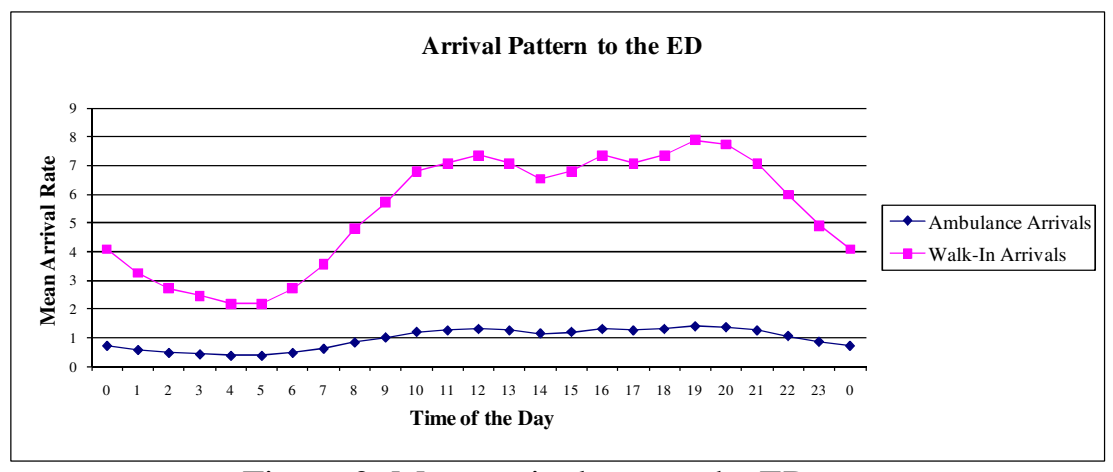

Figure 3: Mean arrival rate to the ED

The same publication used for the arrival rate was used to obtain the percentage of patients that belong to each acuity level. These percentages are shown in Table 1. 
Table 1: Percentages of acuity mix depending on arrival mode.

\begin{tabular}{|c|c|c|c|}
\cline { 2 - 4 } \multicolumn{1}{c|}{} & \multicolumn{2}{c|}{ Arrival Mode } & \\
\hline Acuity Level & Ambulance & Walk-In & Overall \\
\hline 1 & 15 & 2 & 3.95 \\
\hline 2 & 42 & 16 & 19.9 \\
\hline 3 & 30 & 40 & 38.5 \\
\hline 4 & 10 & 30 & 27 \\
\hline 5 & 3 & 12 & 10.65 \\
\hline Overall & 15 & 85 & 100 \\
\hline
\end{tabular}

The treatment time in the ED is assumed to be exponentially distributed with a mean shown in Table 2. The same publication with data from an Arizona provider (Cochran and Roche 2009) was used to derive these times.

Table 2: Mean treatment time depending on acuity level.

\begin{tabular}{cc}
\hline Acuity Level & Mean Treatment Time (min) \\
\hline $\mathbf{1}$ & 261 \\
$\mathbf{2}$ & 261 \\
$\mathbf{3}$ & 162 \\
$\mathbf{4}$ & 90 \\
$\mathbf{5}$ & 30 \\
\hline
\end{tabular}

The percentage of ED patients that require admission to the hospital is assumed to be $15 \%$, which is in the range of admission percentages seen in the literature (CDC 2006; CDC 2008). The inter-arrival time of direct admission to the IP unit and the treatment time in this unit are based on a provider in Arizona (Cochran and Bharti 2006). Direct admissions are assumed to be exponentially distributed with a mean of 10 hours. Treatment times are also assumed to be exponentially distributed with means shown in Table 3.

Table 3: Mean treatment time in the IP unit.

\begin{tabular}{cc} 
Patient Source & Mean Treatment Time in IP (hrs) \\
\hline Direct Admission & 70 \\
Admission from ED & 80 \\
\hline
\end{tabular}

LWOTs are events that occur in real ED's that are complex to model. However, it is important to add it as a feature given its impact on the performance and traffic intensity of an ED. This paper uses an approach employed in other assessments of real systems (Miller, Ferrin and Shahi 2009). If a patient has not been placed in a bed upon 24 hours of arrival, then the patient is removed from the system, representing a LWOT patient.

\subsection{Single-Threshold AD Policies}

Each AD policy studied in this paper considers one out of the three main factors for going on diversion in practice: the number of available inpatient beds, the number of patients waiting and the number of patients boarding. The first and second are the main causes to divert ambulances according to a survey published in the Advance Data from Vital and Health Statistics (CDC 2006). Meanwhile, the last one has been highlighted as another important contributor of diversion (Asplin 2003; Pham et al. 2006). Therefore, the state variables of interest to formulate the policies are the following: 


\section{Ramirez, Fowler and Wu}

$x=$ Total number of patients waiting for a bed in the ED, $x=0,1,2,3, \ldots$

$y=$ Total number of patients boarding in the ED, $y=0,1,2,3, \ldots, B_{\mathrm{ED}}$

$z=$ Number of beds available in the IP unit, $z=0,1,2,3, \ldots, B_{\mathrm{IP}}$

where:

$B_{\mathrm{ED}}=$ Number of beds in the ED. In this paper $B_{\mathrm{ED}}=20$.

$B_{\mathrm{IP}}=$ Number of beds in the Inpatient Unit. In this paper $B_{\mathrm{IP}}=78$.

Single-threshold AD policies have the form: (Don, Doff), where the Don parameter represents the threshold to set the diversion status on and the Doff parameter is the criterion to reevaluate or remove the AD status. Hence, based on this form and the state variables of interests, the six policies studied in this paper are:

P1: $\left(U_{x}, \Delta t\right)$

Where $U_{x}$ is the threshold on the number of patients waiting in the ED to go on diversion. Hence, diversion status is set on if at some point $x \geq U_{x}$. Once the ED has gone on diversion, every $\Delta t$ time units the state of the system is evaluated until the decision to go off diversion is made. Diversion status is removed at a re-evaluation point if $x<U_{x}$.

P2: $\left(U_{x}, L_{x}\right)$

Where $U_{x}$ is the upper threshold on the number of patients waiting in the ED to go on diversion and $L_{x}$ is the lower threshold on the number of patients waiting in the ED to remove the diversion status. Hence, diversion is set on if at some point $x \geq U_{x}$ and it is removed when $x \leq L_{x}$.

P3: $\left(U_{y}, \Delta t\right)$

Where $U_{y}$ is the threshold on the number of patients boarding in the ED to go on diversion. Hence, diversion status is set on if at some point $y \geq U_{y}$. Once the ED has gone on diversion, every $\Delta t$ time units the state of the system is evaluated until the decision to go off diversion is made. Diversion status is removed at a re-evaluation point if $y<U_{y}$.

P4: $\left(U_{y}, L_{y}\right)$

Where $U_{y}$ is the upper threshold on the number of patients boarding in the ED to go on diversion and $L_{y}$ is the lower threshold on the number of patients boarding in the ED to remove the diversion status. Hence, diversion is set on if at some point $y \geq U_{y}$ and it is removed when $y \leq L_{y}$.

P5: $\left(L_{z}, \Delta t\right)$

Where $L_{z}$ is the threshold on the current number of beds available in the Inpatient Unit to go on diversion. Hence, diversion status is set on if at some point $z \leq L_{z}$. Once the ED has gone on diversion, every $\Delta t$ time units the state of the system is evaluated until the decision to go off diversion is made. Diversion status is removed at a re-evaluation point if $z>U_{z}$.

P6: $\left(L_{z}, U_{z}\right)$

Where $L_{z}$ is the lower threshold on the number of beds available in the Inpatient Unit to go on diversion and $U_{z}$ is the upper threshold on the number of beds available in the Inpatient Unit to remove the diversion status. Hence, diversion is set on if at some point $z \leq U_{z}$ and it is removed when $z \geq L_{z}$.

Note that there are two alternatives to re-evaluate the state of the system once the decision of going on diversion has been made. Periodic evaluation implies that the state of the system will be reviewed only at discrete times, such as P1, P3 and P5. On the other hand, continuous review monitors the state of the system at every event in the ED, such as P2, P4 and P6. 


\subsection{Experimental Design}

Based on the policies described in Section 3.3, the levels for each policy are defined with the purpose of covering a wide range of values, which include conservative and not so conservative policies. The levels are shown in Table 4. The experimentation is based on a general factorial design for each policy, where all the feasible combinations of instances are chosen as treatment for experimentation (Montgomery 2005). Note that in the case of P2 and P4, it is required that Don > Doff; meanwhile for P6, Don < Doff is required.

Table 4: Levels of the policy parameters chosen for experimentation.

\begin{tabular}{ccc} 
Policy & \multicolumn{1}{c}{ Don } & Doff \\
P1 & $10,20,30,40,50,60,70$ patients & $15,30,45,60$ minutes \\
P2 & $10,20,30,40,50,60,70$ patients & $0,10,20,30,40,50,60$ patients \\
P3 & $1,2,3,4,5,6,7,8,9,10$ patients & $15,30,45,60$ minutes \\
P4 & $1,2,3,4,5,6,7,8,9,10$ patients & $0,1,2,3,4,5,6,7,8,9$ patients \\
P5 & $0,1,2,3,4,5,6,7,8,9$ beds & $15,30,45,60$ minutes \\
P6 & $0,1,2,3,4,5,6,7,8,9$ beds & $1,2,3,4,5,6,7,8,9,10$ beds
\end{tabular}

The simulation model is adapted for every policy and every value combination to collect data regarding patient waiting time and time spent on diversion. A pilot run showed that three weeks of warm up period was acceptable and then thousands of observations are collected per replicate. Forty replications are run using antithetic random variates (Law 2007), obtaining twenty observations per treatment.

\section{ANALYSIS OF RESULTS}

\subsection{Mean Performance Across the Policies}

The mean performance for all the treatments across the six policies are presented in Figure 4. It can be seen that the band comprising the solutions including all the policies resembles a piecewise linear graph, whose first segment includes solutions belonging to P1, P2, P3 and P4, while the second segment includes solutions of P5 and P6.

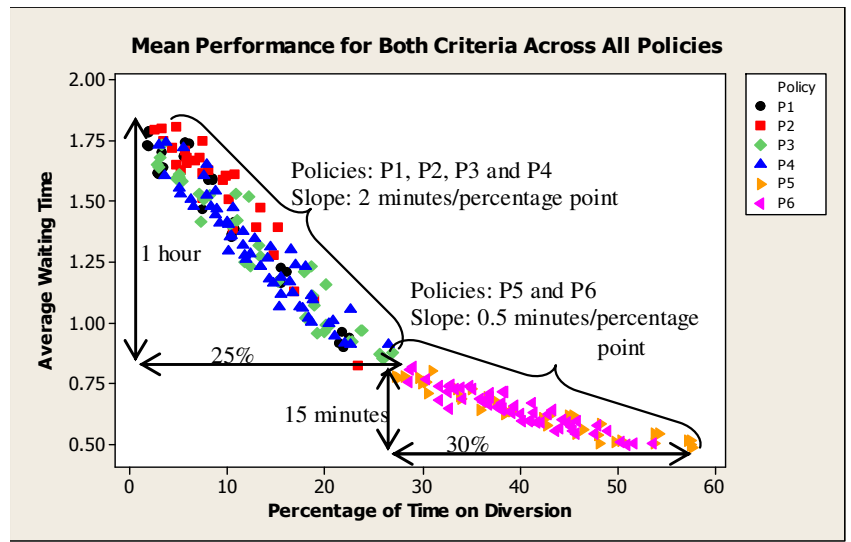

Figure 4: Bi-criteria graph with solutions across all the policies

Policies based on the number of patients waiting (P1 and P2) and the number of patients boarding (P3 and $\mathrm{P} 4)$ produce results that yield the lowest proportion of time spent on diversion. In addition, they have the highest gaining in the trade-off between accessibility and service; thus for every percentage point increase in diversion status, the average waiting time is reduced about two minutes. 
On the other hand, the policies based on the availability of IP beds (P5 and P6) seem to be the most conservative policies, yielding a small average waiting time, but spending a high percentage of time on diversion status. Considering that the most common reason to go on diversion is the lack of IP beds available (CDC 2006), these results could explain the concerns of the large number of hours diverting ambulances in the real systems.

In addition, the policies based on the same factor are compared using bi-criteria graphs. Figure 5 shows the graph for P1 and P2, which are based on the number of patients waiting and have periodic and continuous review, respectively. It can be seen that results with a common Don parameter form a cluster in the case of periodic review, which means that the time to re-evaluate the state of the system is not a significant parameter. On the other hand, the clusters using continuous review overlap each other.

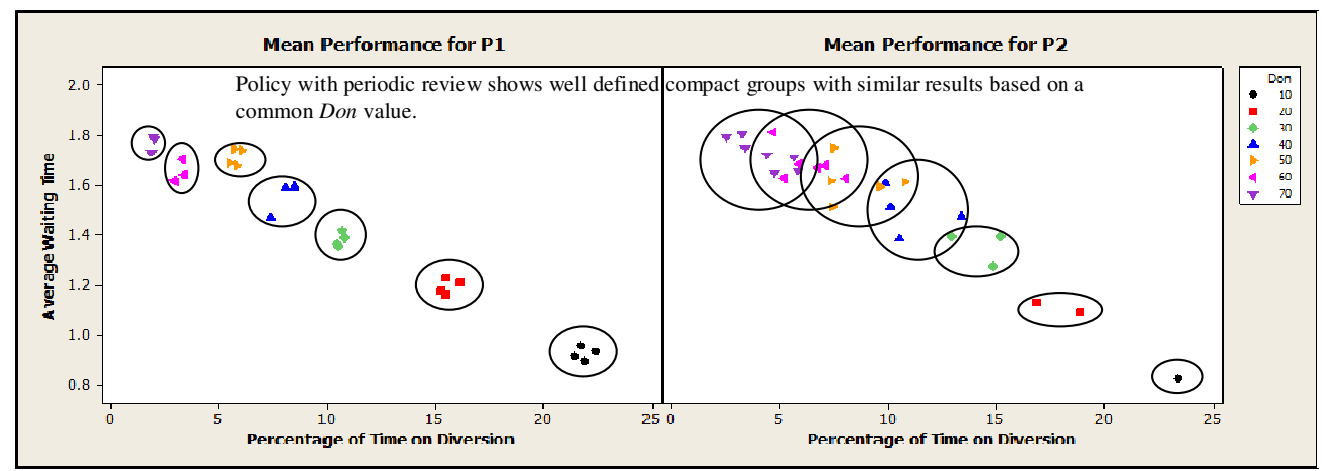

Figure 5: Bi-criteria graphs of the mean performance for P1 and P2

The conclusions of the comparison between P3 and P4, as well as between P5 and P6 are very similar. The clustering of solutions based on a common Don value is also evident in the case of periodic review for those policies. However, the dispersion of the solutions increases for continuous review. For a more complete analysis of these graphs please refer to Ramirez et al. (2010b).

In summary, the analysis of the mean performance in the bi-criteria evaluation of the six policies lead to the following observations:

- There is a trade-off between time spent on diversion and average waiting time when AD is implemented.

- Policies based on number of patients waiting and number of patients boarding offer a good balance between accessibility to emergency care and average waiting time.

- In addition, these policies lead to larger reductions of average waiting time per every percentage point spent on diversion.

- Even though the policies based on availability of IP beds produce a very small average waiting time, they yield a high percentage of time spent on diversion, which is very likely to be undesirable by the decision makers.

- The periodic review of the system produce clusters of solutions with a common value for the Don parameter, implying that Doff parameter is not significant.

\subsection{Variability Across the Policies}

The mean performance is important for decision makers to decide what is the best policy that balances average waiting time and time spent on diversion according to the needs of the organization. However, the consistency of the policies is not included in previous graphs. Therefore, this section evaluates the variability across the six policies. 


\section{Ramirez, Fowler and Wu}

Confidence intervals constructed for each criterion yield a good relative precision across the six policies. For instance, relative precision of $95 \%$ confidence intervals on the average waiting time is between $5.8 \%$ and $7.1 \%$ across the six policies. For the percentage of time on diversion, the precision is between $5.09 \%$ and $12.88 \%$. These percentages imply a high chance of covering the true value; however, they do not provide much information about the differences across the policies.

Since the purpose of this paper is to analyze both criteria simultaneously, 95\% confidence ellipses that jointly include the average waiting time and percentage of time on diversion are constructed and presented in Figure 6. Note that each confidence ellipse is built for every treatment considering all its replications. Remarkable differences can be seen from these graphs comparing all the policies.

In the first place, it is evident the change of the ellipse depending on the factor considered in the policy. Thus, policies based on the number of patients waiting have a well defined ellipse with a smaller area than those based on the number of patients boarding. Furthermore, P1 exhibits ellipses that overlap each other for common values of the Don parameter, which implies that Doff is not significant for this policy. Policies based on the number of patients boarding also show a high correlation between the two variables; however, there are more ellipses overlapping, making it difficult to decide what policies could produce better results for the decision maker.

On the other hand, ellipses for policies based on availability of IP beds have their major axes almost parallel to the horizontal axes. This implies that correlation between the two criteria is weak; hence the prediction of performance becomes difficult because results are randomly distributed.

Thus, the main observations in terms of the variability in the performance that each policy produces are:

- Policies based on the number of patients waiting and number of patients boarding have a higher correlation between the two criteria evaluated on this paper. This can increase the accuracy of the prediction of performance under a specific AD policy.

- On the contrary, policies based on the number of available beds in the IP unit have a low correlation between the two criteria.

- Policies based on number of patients waiting have smaller areas in the ellipses; thus, they are more precise. In addition, P1 confirms that Doff parameter is not significant for this policy.

\section{CONCLUSIONS}

Overcrowding of ED's across the United States has been a concern for many people. Diverse actions exist to relieve congestion from ED's and one of them is AD. AD has been widely discussed by the medical community for the potential effects of delaying treatment. However, there is a lack of applying modeling tools to analyze the impact of $\mathrm{AD}$. This paper proposes a methodology that graphically evaluates the trade-off between time spent on diversion and average waiting time for patients in the ED.

Even though the policies studied using a single threshold may be simple, the findings can be used to explain the behavior seen in real systems, given that most AD decisions are made based primarily on a single factor in reality. Thus, the insights gained from this study can be used for AD decisions for improved quality of care. Future extensions of this research include the use of Genetic Algorithms to find the form of the policies that yield the best results considering multiple criteria. In addition, Approximate Dynamic Programming will be used to assess the diversion decision in order to minimize the non-value added time in a multi-hospital system. 


\section{Ramirez, Fowler and $\mathrm{Wu}$}
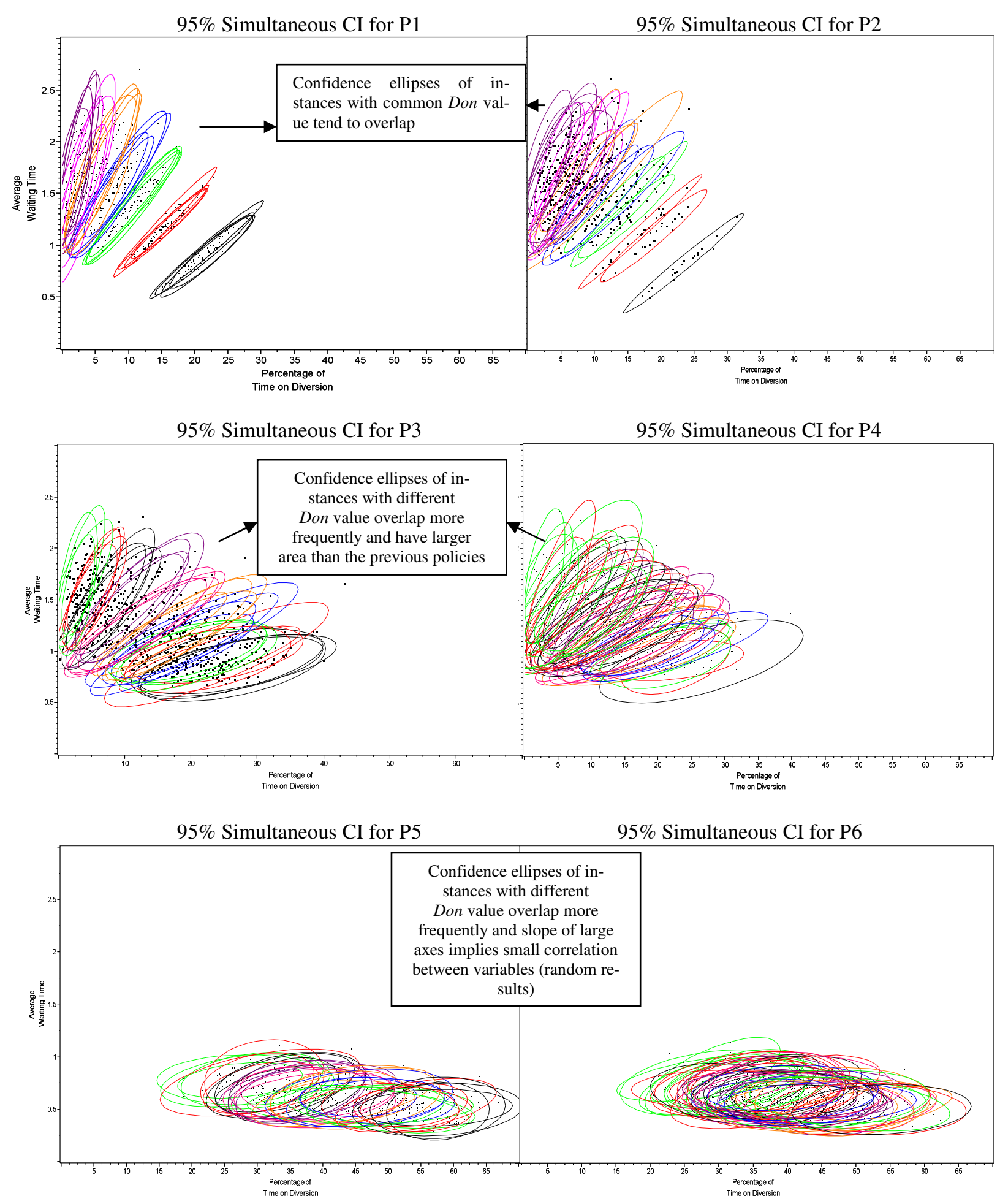

Figure 6: 95\% Simultaneous confidence intervals 
Ramirez, Fowler and Wu

\section{REFERENCES}

Asplin, B.R. 2003. Editorial: Does Ambulance Diversion Matter?, Annals of Emergency Medicine 41: 477-480.

Carlyle, W.M., J.W. Fowler, E.S. Gel, and B. Kim. 2003. Quantitative Comparison of Approximate Solution Sets for Bi-criteria Optimization Problems, Decision Sciences 34:63-82.

Centers for Disease Control and Prevention. 2006. Staffing, Capacity, and Ambulance Diversion in Emergency Departments: United States, 2003-04, Advance Data from Vital and Health Statistics 376.

Centers for Disease Control and Prevention. 2008. National Hospital Ambulatory Medical Care Survey: 2006 Emergency Departments Summary, National Health Statistics Reports 7.

Cochran, J.K., and A. Bharti. 2006. A Multi-Stage Stochastic Methodology for Whole Hospital Bed Planning under Peak Loading, International Journal of Industrial and Systems Engineering 1:8-36.

Cochran, J.K., and K.T. Roche. 2009. A Multi-Class Queuing Network Analysis Methodology for Improving Hospital Emergency Department Performance, Computers \& Operations Research 36:14971512.

Hagtvedt, R., P. Griffin, P. Keskinocak, M. Ferguson and F.T. Jones. 2009. Cooperative Strategies to Reduce Ambulance Diversion, In Proceedings of the 2009 Winter Simulation Conference, ed. M.D. Rossetti, R.R. Hill, B. Johansson, A. Dunkin, and R.G. Ingalls, 1861-1874. Piscataway, New Jersey: Institute of Electrical and Electronics Engineers, Inc.

Kolker, A. 2008. Process Modeling of Emergency Department Patient Flow: Effect of Patient Length of Stay on ED Diversion, Journal of Medical Systems 32:389-401.

Law, A.M. 2007. Simulation Modeling \& Analysis. $4^{\text {th }}$ ed. McGraw-Hill, Inc.

Massachusetts Nurse Newsletter. 2009. State's' no diversion policy' is putting strain on Massachusetts hospitals. April 2009:8-9

Miller, M., D. Ferrin, and N. Shahi. 2009. Estimating Patient Surge Impact in Several Regional Emergency Departments, In Proceedings of the 2009 Winter Simulation Conference, ed. M.D. Rossetti, R.R. Hill, B. Johansson, A. Dunkin, and R.G. Ingalls, 1906-1915. Piscataway, New Jersey: Institute of Electrical and Electronics Engineers, Inc.

Montgomery, D.C.2005. Design and Analysis of Experiments. $6^{\text {th }}$ ed. John Wiley \& Sons, Inc.

Pham, J.C., R. Patel, M.G. Millin, T.D. Kirsch and A. Chanmugam. 2006. The Effects of Ambulance Diversion: A Comprehensive Review, Journal of the Academic Emergency Medicine 13:1220-1227.

Ramirez, A., J.W. Fowler, and T. Wu. 2009. Analysis of Ambulance Diversion Policies for a Large-Size Hospital, In Proceedings of the 2009 Winter Simulation Conference, ed. M.D. Rossetti, R.R. Hill, B. Johansson, A. Dunkin, and R.G. Ingalls, 1875-1886. Piscataway, New Jersey: Institute of Electrical and Electronics Engineers, Inc.

Ramirez, A., J.W. Fowler, E.S. Gel, and T. Wu. 2010a. Analysis of Min-Max Ambulance Diversion Policies Using Queuing Theory, In Proceedings of the 2010 Industrial Engineering Research Conference, ed. A. Johnson and J. Miller.

Ramirez, A., J.W. Fowler, and T. Wu. 2010b. Bi-Criteria Analysis of Single-Threshold Ambulance Diversion Policies. Manuscript.

Springer's International Series. 2006. Patient Flow: Reducing Delay in Healthcare Delivery. Edited by Randolp W. Hall.

Tan, P.-N., M. Steinbach and V. Kumar. 2006. Introduction to Data Mining. $1^{\text {st }}$ ed. Pearson Education, Inc.

United States General Accounting Office. 2003. Hospital Emergency Departments: Crowded Conditions Vary among Hospitals and Communities. Report to the Ranking Minority, Committee on Finance, U.S. Senate. 


\section{AUTHOR BIOGRAPHIES}

ADRIAN RAMIREZ is a PhD student in Industrial Engineering in the School of Computing, Informatics and Decision Systems Engineering at Arizona State University. His research interests include modeling, simulation and analysis of healthcare delivery systems. He received a MS in Manufacturing Systems at ITESM and a BS in Industrial Engineering at Universidad de Sonora, both in Mexico. His email address is <adrian.ramirezeasu.edu>.

JOHN W. FOWLER is a Professor in the Operations Research and Production Systems group of the School of Computing, Informatics and Decision Systems Engineering at Arizona State University. His research interests include modeling, analysis, and control of manufacturing and service systems. He is a Fellow of the Institute of Industrial Engineers and is the SCS representative on the Board of Directors of the Winter Simulation Conference. He is an Area Editor of the Transactions of the Society for Computer Simulation International, an Associate Editor of IEEE Transactions on Semiconductor Manufacturing, and will be the Editor of a new journal entitled IIE Transactions on Healthcare Systems Engineering. His email address is $<$ john. fowler dasu. edu $>$.

TERESA WU is an Associate Professor of the School of Computing, Informatics and Decision Systems Engineering at Arizona State University. She received her Ph.D. in Industrial Engineering from the University of Iowa in 2001. Her current research interests include: distributed decision support, distributed information system, healthcare informatics and data fusion.. Professor $\mathrm{Wu}$ has over 40 articles published (or accepted) in such journals as International Journal of Production Research, Information Science, IEEE Transactions on Engineering Management, ASME: Journal of Computing and Information Science in Engineering. She serves on the Editorial Review Board for International Journal of Production Research, IEEE Transactions on Engineering Management, Computer and Standard Interface, International Journal of Electronic Business Management. Her email address is <teresa . wu@asu.edu>. 